\title{
Correspondence
}

\section{Modified retrobulbar block}

To the Editor:

We read with interest the recently published article by Drs. Wong, Koehrer and Sutton' introducing a modified retrobulbar block for eye surgery. A technique of ophthalmic regional anaesthesia should be based on sound anatomical principles, especially in the selection of a needle path and depth of insertion that will avoid structures such as important blood vessels, extraocular muscles and the optic nerve. In addition, it should include precise instruction as to direction of needle insertion. We have analysed the technique described in the article and suggest that it falls short in several of these areas and may be prone to the production of serious complications, such as globe perforation, optic nerve trauma, intraorbital haemorrhage or injection into one of the extraocular muscles with resultant local anaesthetic myotoxity.

It is argued that, if a retrobulbar needle can be placed in a straight line rather than with redirection after partial orbital insertion, perforation of the globe is less likely. Since the globe is closer to the orbit roof than its floor ${ }^{2}$ there is more reason for needle redirection with performing retrobulbar injection from above rather than from below the globe.

In the described modified retrobulbar technique the needle is advanced in the sagittal plane but no instruction is given regarding the angle of insertion. From illustration $\mathrm{C}$, the needle path appears to be on the medial side of the superior rectus/levator palpebae complex yet on the lateral side of the optic nerve. This is anatomically impossible for a needle advancing in a sagittal plane. With the description of the needle path and insertion to 32-33 $\mathrm{mm}$ it is possible to be in line with the optic nerve which is a more medial orbital structure than is commonly realized, particularly towards the orbit apex.

We believe selection of a superomedial needle entry point is ill-advised not only because of the trochlear complex, although it may well be lateral to the described path, but also because this quadrant is the most vascular part of the anterior orbit, containing the end arteries of the ophthalmic arterial system. In addition, the superior ophthalmic vein, the largest venous channel of the orbit, courses anteromedially to posterolaterally, in the orbit between the superior rectus muscle and the optic nerve, across the described path of the needle. Furthermore, we are concerned that the needle avoid the medial rectus and superior oblique muscles along the pathway described.

Finally, the depth of needle entry into the orbit described both for the standard retrobulbar and the modified retrobulbar blocks are in excess of the $31 \mathrm{~mm}$ amount recommended as maximum orbit penetration by Katsev et al. in their extensive analysis of safe practice based on bony anatomy. ${ }^{3}$

Robert C. Hamilton MB BCh FRCPC

Rock G. Loken BSc MD FRCPC

Department of Anaesthesia

The University of Calgary

\section{REFERENCES}

1 Wong $D H W$, Koehrer E, Sutton HF. A modified retrobulbar block for eye surgery. Can J Anaesth 1993; 40: 547-53.

2 Grizzard WS. Ophthalmic anesthesia. In: Reinecke RD (Ed.). Ophthalmology Annual. New York: Raven Press: 1989: 265-94.

3 Katsev DA, Drews RC, Rose BT. An anatomic study of retrobulbar needle path length. Ophthalmology 1989; 96 : 1221-4.

\section{$R E P L Y$}

I thank Drs Hamilton and Loeken for their interest in our paper, and apologize for the lack of detail in the description of our technique, and the somewhat misleading perspective of the accompanying illustrations. The needle is inserted through the upper eyelid, between the roof and the medial wall at 10 to 11 o'clock position (for the left eye), well clear of the upper orbital margin and the trochlea. It is advanced slowly downwards at a plane $10^{\circ}$ to the floor of the orbit and with a very slight temporal tilt from the sagittal plane. There should be no resistance to the passage of the needle. The needle remains straight throughout its course. With no redirection around the equator of the globe, it passes below the trochlea and superior oblique/levator palpebrae complex, and enters the muscle cone between the medial and superior rectus muscles. The course of the needle was demonstrated in cadaver dissections before undertaking the study, and again before writing this reply.

Katsev et al. recommended, in the abstract of their paper, that needles be placed less than $31 \mathrm{~mm}$ (1.25 in) behind the orbital rim. Their study on 120 orbits showed a considerable variation of the retrobulbar needle path length (from the junction of the middle and lateral third of the infraorbital rim to the superior nasal aspect of the optic foramen) of 42 to 54 $\mathrm{mm}$, due to racial or sexual differences. The needles used for retrobulbar injection at their institution ranged from 31.5 to $50 \mathrm{~mm}$ in length. The authors stated that "it is this variation that may put patients with smaller orbits at greater risk if 35- 
mm or longer needles are used." They also stated that "with a 35-mm needle, piercing the optic nerve sheath is less likely than with a 38-mm needle, but a $31.5-\mathrm{mm}$ needle is still safer." Obviously, common sense prevails as to the total length to be inserted for different sizes of orbits.

The presence of the superior ophthalmic vein and its two roots are quite constant in the supero-medial quadrant of the anterior orbit. The inferior ophthalmic vein is more variable on the orbital floor. Nowhere in the orbil is entirely free from arteries and veins. This is why haemorrhage can complicate retrobulbar and even peribulbar injections.

It is hard to break from tradition, even from a technique which ofien results in suboptimal akinesia, and has produced many reports of retrobulbar haemorrhage, globe perforation, brainstem anaesthesia, and extraocular muscle injury. Ohr modified technique is simple to administer, does not require additional facial nerve block, and produces good akinesia. $I t$ is proposed as an alternative technique and not as a replacement of the classical retrobulbar block. I have used it in over 600 patients, without any serious complications. However, as with the classical technique, no one can guarantee that these would not occur

David H.W. Wong MB BS FRCPC

Department of Anaesthesia

University of British Columbia

Vancouver

\section{Confirmation of internal jugular vein cannulation: the Finucane technique}

To the Editor:

We wish to report a method of confirming internal jugular vein cannulation which was developed at Grady Memorial Hospital in 1986. This technique is currently being used at the University of Wisconsin and Emory University and both faculty and staff have used this technique in over 3,000 cases.

The internal jugular vein is the most frequent site for central venous cannulation by anaesthetists, and has a low incidence of complications. The most frequent complication is carotid artery puncture, which has an incidence of $2 \%$ in most series. ${ }^{1,2}$ This can, if not recognized, lead to loss of the airway from bleeding or neurological insult. Methods to avoid major complications must be employed. Commonly, internal jugular vein cannulation is confirmed prior to the insertion of large cannulas, i.e., $8.5 \mathrm{Fr}$ introducers, by connecting the intravenous catheter to pressure tubing and electronic transducer and observing a central venous wave form. ${ }^{3}$ However, transducers are not always available. We describe an alternative method for confirmation of internal jugular vein cannulation.

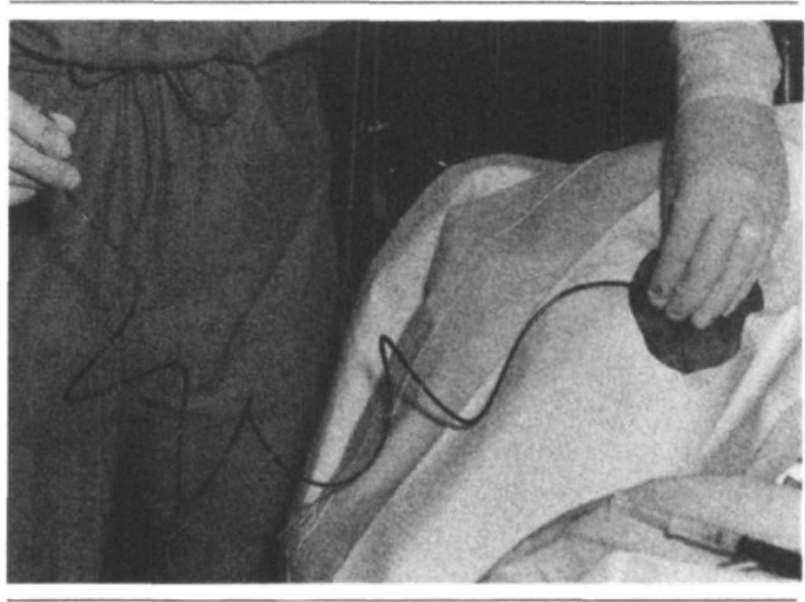

FIGURE Sterile pressure tubing has been attached to an 18 gauge angiocath. The pressure tubing has been placed in a dependent position, allowing it to fill with venous blood.

After cannulation of the internal jugular vein with either an 18 or 20 gauge catheter, a $24^{\prime \prime}$ length of sterile tubing is connected directly to the catheter. The open end of the tubing is lowered, allowing it to refill freely with blood (Figure). The open end is then raised, allowing the blood to run freely into the vein. We commonly use oxygen during central vein cannulation and monitor the patient with pulse oximetry to enhance the contrast between the venous and arterial blood. ${ }^{4}$ The arterial venous colour difference can be a useful clinical guide but must be used in the context of clinical circumstances.

We contend that five observations will confirm intravenous placement: (1) non-pulsatile filling of the tubing; (2) a dark venous colour of the blood, particularly when compared with a simultaneous arterial sample and the patient is breathing oxygen; (3) an inability to fill the tubing completely when held vertically; (4) a smooth decrease in the height of the column of blood when the tubing is raised vertically - indicating that the catheter is in a low-pressure system and is neither kinked nor against a vessel wall; (5) variation of the column of blood with respiration or a valsalva manoeuvre,

Although no method will completely prevent unintentional cannulation of the carotid artery, we feel that this technique is valuable when transducers are not available, or when working alone or involved in an emergency internal jugular cannulation.

George A. Amdt MD*

Thomas Felton MD* $^{*}$

Brendan Finucane MD $\ddagger$

Albert Santora MD $\dagger$

Department of Anesthesiology

*University of Wisconsin Clinical Science Center,

Madison, Wisconsin 